\title{
Sonographic Assessment of Spleen Stiffness Before and After Transjugular Intrahepatic Portosystemic Shunt Placement With or Without Concurrent Embolization of Portal Systemic Collateral Veins in Patients With Cirrhosis and Portal Hypertension
} A Feasibility Study

Paula M. Novelli, MD, Kyung Cho, MD, FSIR, Jonathan M. Rubin, MD, PhD

Received April 22, 2014, from the Department of Radiology, Divisions of Vascular and Interventional Radiology (P.M.N., K.C.) and Abdominal Imaging (J.M.R.), University of Michigan Health System, Ann Arbor, Michigan USA. Revision requested May 8, 2014. Revised manuscript accepted for publication June 23, 2014.

Address correspondence to Paula M. Novelli, MD, Department of Radiology, Division of Vascular and Interventional Radiology, University of Michigan Health System, 1500 E Medical Center Dr, UHB1D530, Ann Arbor, MI 48109 USA.

E-mail:paulanov@umich.edu

Abbreviations

TIPS, transjugular intrahepatic portosystemic shunt

doi:10.7863/ultra.34.3.443
Objectives - To determine the feasibility of spleen stiffness measurement in the evaluation of portal hemodynamics in patients undergoing transjugular intrahepatic portosystemic shunt (TIPS) placement.

Methods -We prospectively correlated the spleen stiffness as measured by the shear wave velocity with the portal pressure and portosystemic gradient in patients undergoing TIPS procedures. Twenty-three consecutive patients referred for placement of a TIPS were enrolled. Included in our study were 19 patients in whom a spleen stiffness measurement was obtained before, immediately after, and 1 to 3 days after placement. Spleen stiffness was measured by calculating the Young modulus estimated from the shear wave velocity. A 2-tailed nonparametric Mann-Whitney $U$ test was used to assess statistically significant differences in spleen stiffness measurement after TIPS placement, and regression analysis was used to correlate spleen stiffness measurement with portal pressure.

Results-After TIPS placement, the spleen stiffness measurement increased, with a mean increase in the Young modulus \pm SD of $6.54 \pm 6.29 \mathrm{kPa}$ in $42 \%$ of patients ( 8 of 19). In the remaining 58\% (11 of 19), the spleens became softer after TIPS placement (Young modulus decreased by $9.57 \pm 8.82 \mathrm{kPa}$ ). Eight patients, including 5 with concurrent embolization or thrombosis of competitive shunts, had increased spleen stiffness. The mean change in the median spleen stiffness before and after TIPS placement between the patients with and without competitive shunts was statistically significantly different $(P<.04$, nonparametric Mann-Whitney $U$ test $)$. There was no measurable correlation between spleen stiffness measurement and portal pressure before and after TIPS placement.

Conclusions - This study demonstrates the feasibility of a noninvasive spleen stiffness measurement, which could complement conventional sonography with additional functional information in patients undergoing TIPS procedures.

Key Words - gastrointestinal ultrasound; portal hemodynamics; portal hypertension; sonography; spleen stiffness; transjugular intrahepatic portosystemic shunt; variceal bleeding 
$\mathrm{P}$ ortal hypertension and portosystemic collateral veins develop due to increased intrahepatic resistance to portal blood flow in cirrhosis of the liver. ${ }^{1}$ The spectrum of physiologic abnormalities occurring from portal hypertension includes ascites, variceal hemorrhage, and splenomegaly. These clinical sequelae and the portal circulation are evaluated by using various clinical and laboratory tests and radiographic studies, including liver function studies, endoscopy, noninvasive imaging modalities (sonography, computed tomography, and magnetic resonance imaging), and angiography with hepatic manometry. ${ }^{2}$

The portal venous system is a complex circuit made up of the liver, the spleen, and splanchnic venous tributaries, including portosystemic collaterals and varices. Splenic pulp pressure obtained during splenoportography reflects portal pressure in patients with both postsinusoidal and presinusoidal blocks. ${ }^{3}$ We hypothesized that sonographic assessment of spleen stiffness would give a noninvasive measurement of splenic pulp pressure and portal pressure and thus would predict transjugular intrahepatic portosystemic shunt (TIPS) patency. Therefore, we performed spleen stiffness measurement before and after TIPS placement in 19 patients with cirrhosis and portal hypertension.

\section{Materials and Methods}

Our Institutional Review Board approved this study protocol, and patients provided written informed consent. We enrolled 23 consecutive patients with cirrhosis and portal hypertension who were referred for a TIPS or shunt revision between September 2011 and June 2012. Of the enrolled patients, 19 were analyzed in this study, and 4 were excluded. We were unable to place a TIPS in 2 of these patients. Two of the excluded patients did not have data obtained after TIPS placement. One enrolled patient did not have cirrhosis. The indications for TIPS placement included variceal bleeding $(\mathrm{n}=6)$, intractable ascites/ hepatic hydrothorax $(n=9)$, and both $(n=4$; Table 1$)$.

\section{Technique}

A physician with 35 years of experience in sonography oversaw all sonographic examinations. We used an iU22 ultrasound scanner (Philips Healthcare, Bothell, WA) with shear wave generation capability to measure spleen stiffness by a calculated Young modulus estimate based on the shear wave velocity (Figure 1). The relationship in a linear elastic isotropic medium is $\mu=\rho v 2$, where $\mu$ is the shear elastic modulus; $\rho$ is the density of the medium; and $v$ is the shear wave speed. For an incompressible medium, $E=3 \mu$, where $E$ is the Young modulus. ${ }^{4}$ Therefore, tracking shear wave speed can provide the means to calculate the shear elastic modulus and the Young modulus.

Measurements were obtained with a C5-1 curved linear transducer. In the shear wave velocity mode, the operator places a defined region of interest within the 2dimensional grayscale image. At that position, the machine launches a high-intensity (still within US Food and Drug Administration limits) ultrasonic pulse, which locally displaces the tissue at that position. A shear wave is generated, and the machine then tracks the propagation of the shear wave normal to the propagation direction of the highintensity pulse. Eight to 15 measurements were obtained in each acquisition period. Pre-TIPS data were collected within 1 hour of the TIPS procedure. In the procedure room immediately after completion of the TIPS procedure, the second set of data was obtained. Additional data were obtained in a nonfasting state on day 1 in all analyzed patients. Patients remaining hospitalized on days 2 and 3

Table 1. Patient Demographics

\begin{tabular}{lc}
\hline Characteristic & Value \\
\hline Etiology of cirrhosis, $\mathrm{n}$ & \\
Ethanol & 8 \\
Hepatitis C & 2 \\
Nonalcoholic steatohepatitis & 3 \\
Cryptogenic & 4 \\
Other & 2 \\
Male/female, $\mathrm{n}$ & $13 / 6$ \\
Mean age (range), y & $54(22-79)$ \\
Indication for TIPS, $\mathrm{n}$ & \\
Ascites/hydrothorax & 9 \\
Variceal bleeding & 6 \\
Both & 4 \\
\hline
\end{tabular}

Figure 1. Sonogram of the spleen obtained during shear wave velocity measurement. The spleen stiffness measurement was recorded at a depth of less than $6 \mathrm{~cm}$ from the skin.

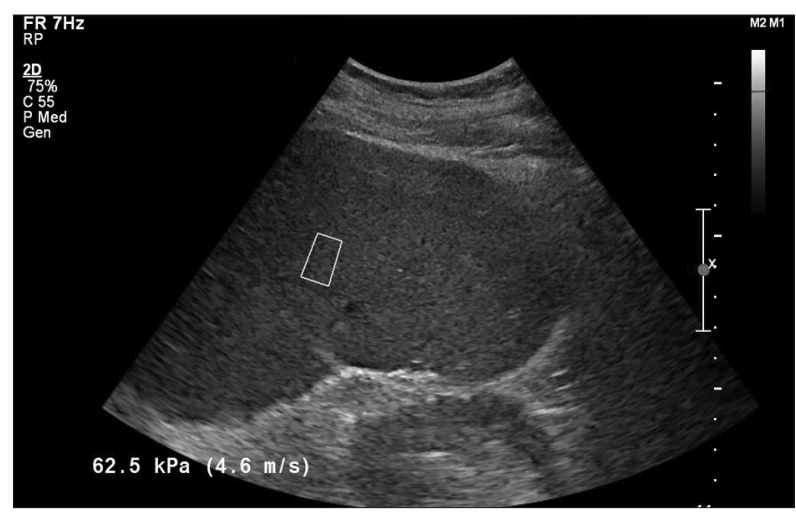


after the procedure had an additional data set each day. Measurements were obtained in the supine position with the ultrasound transducer positioned over the ribs. With this intercostal approach, there was no direct contact between the surface of the probe and the spleen. A preload from pressure applied by the transducer was, therefore, not an issue. Each data set consisted of 8 to 15 measurements obtained throughout the spleen with a breath-holding technique. Care was taken to avoid obtaining measurements in areas with visible vessels or calcified granulomas. The maximum depth evaluable by our system was $6 \mathrm{~cm}$. We then used the median of each series of measurements in the subsequent analysis. Figure 2 shows a series of box plots displaying the distributions of spleen modulus estimates in a single patient from immediately after TIPS placement to 3 days after placement. These show the data spread in a series of typical acquisitions.

After placement of a TIPS between the right hepatic vein and right portal vein using a 10-mm-diameter Viatorr TIPS endoprosthesis (W. L. Gore \& Associates, Flagstaff, AZ), a 5F Omni Flush catheter (AngioDynamics, Latham, $\mathrm{NY}$ ) was placed in the proximal splenic vein for measurement of the portal pressure and direct splenoportography. The portosystemic gradient was determined by subtracting the right atrial pressure from the portal pressure. In 4 of the

Figure 2. Distribution of the modulus measurements made on successive occasions in patient 19 from immediately after TIPS placement to 3 days after placement. The crosses above the plots represent outlier measurements lying beyond the $75 \%$ percentile $+1.5 \times$ interquartile range.

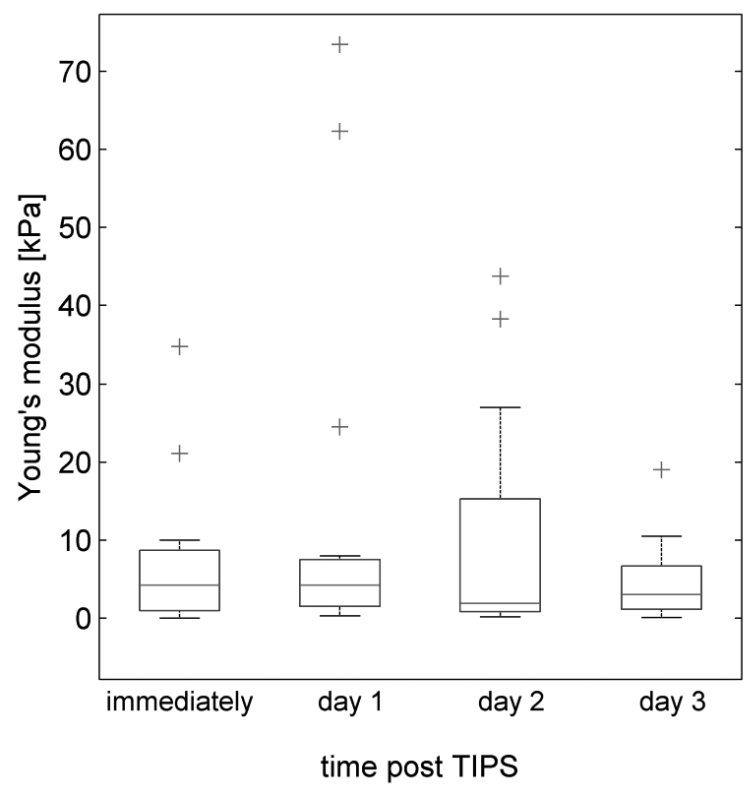

patients, portosystemic collateral veins were embolized with coils (Cook Medical, Inc, Bloomington, IN), dehydrated alcohol injection, USP (Akorn, Inc, Lake Forest, IL), and Amplatzer vascular plugs (St Jude Medical, St Paul, MN).

\section{Statistical Analysis}

The statistical analysis was performed with Excel Analysis ToolPak 2010 (Microsoft Corporation, Redmond, WA) for linear regression analysis and SocialStatistics (www.sociscistatistics.com) for a 2-tailed nonparametric Mann-Whitney U test of the means of the median measurements. The box-and-whisker plots were created with MATLAB (The MathWorks, Inc, Natick, MA). A threshold of $P<0.05$ was used to determine statistical significance.

\section{Results}

In all 19 patients, TIPS placement decompressed the portal system, bringing the portosystemic gradient to less than $12 \mathrm{~mm} \mathrm{Hg}$. The shunt became an outflow tract from the liver and portal venous system (Figure 3). The direction of the splenic and portal vein blood flow was hepatopetal toward the shunt. Portosystemic collateral veins visualized before TIPS placement continued to fill after placement. Embolization successfully occluded the competitive portosystemic varices in 4 patients in whom it was attempted (Figure 4).

In $8(42 \%)$ of the 19 patients, the spleen stiffness increased, with a mean increase in the Young modulus \pm SD of $6.54 \pm 6.29 \mathrm{kPa}$ after TIPS placement. Five of these 8 patients had either undergone concurrent embolization of the associated competitive portosystemic collaterals or had competitive shunts that spontaneously thrombosed. In the remaining 11 patients (58\%), the spleen became softer after TIPS (Young modulus decreased by $9.57 \pm$ $8.82 \mathrm{kPa}$ ). There was no measurable correlation between spleen stiffness and portal venous pressures (Figure 5). The pressure gradient did not correlate with the median modulus change before and after TIPS placement (Figure 6). There was a statistically significant change in the spleen modulus after TIPS placement between the group with embolized or spontaneously thrombosed competitive shunts and the group without shunts (Figure $7 ; P<.04$ ). After TIPS placement, the shunt became the main outflow tract from the liver and portal venous system. The direction of splenic venous blood flow was hepatopetal before and after TIPS placement. 


\section{Discussion}

The role of a TIPS in the management of ascites and variceal hemorrhage is well established. ${ }^{5}$ Good long-term clinical outcomes in these patient populations are influenced by close sonographic shunt surveillance for mainte-

Figure 3. Splenoportograms before (A) and after (B) shunt placement. A, Portogram from the transjugular approach showing a patent portal vein with hepatopetal portal blood flow. B, Portogram after TIPS placement. Most of the contrast medium flows through the TIPS. After TIPS placement, the blood flow in the right hepatic portal vein has reversed from its normal direction toward the TIPS stent.

A

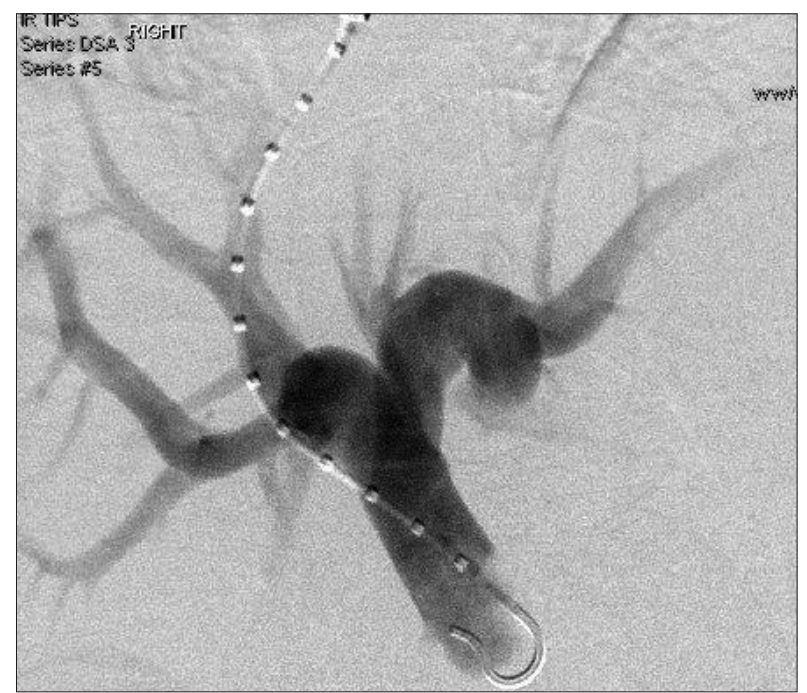

B

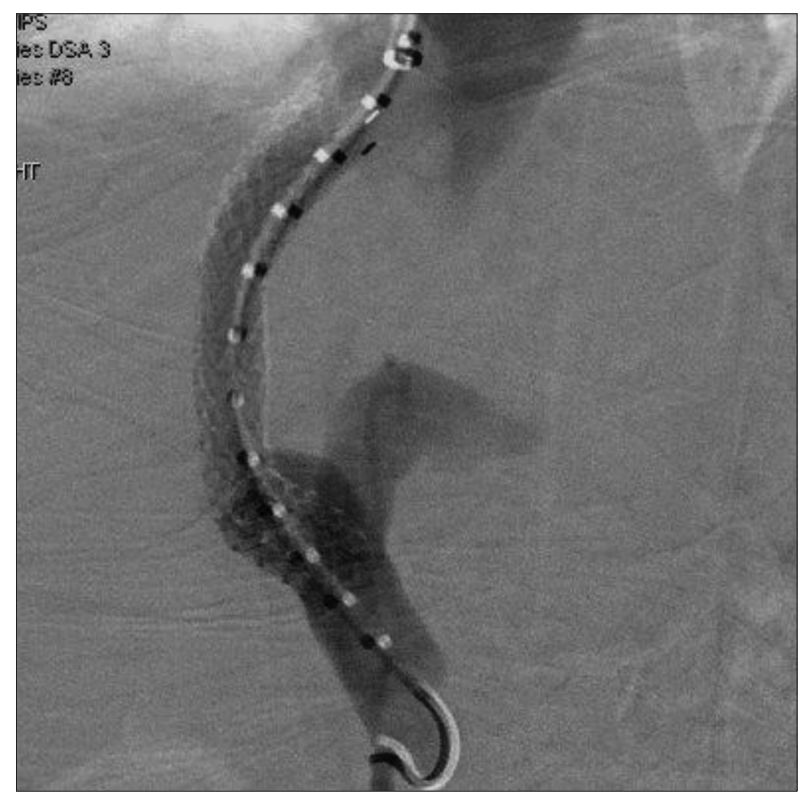

nance of patency. ${ }^{6}$ We hypothesized that a noninvasive assessment of spleen stiffness may have useful predictive value in determining the status of the TIPS. Ultrasound elastography is a method of imaging tissue hardness. A form of elastography is known as shear wave speed imaging. Because the splenic pulp pressure reflects the portal pres-

Figure 4. Embolization of a competitive shunt during a TIPS procedure. A, Splenoportogram before TIPS placement. The short gastric veins from the splenic vein and the left gastric vein from the portal vein feed the gastric fundal varices, which drain via the inferior phrenic vein into the left renal vein and then toward the inferior vena cava. B, Portogram with injection of contrast medium into the superior mesenteric vein after TIPS placement with coil embolization of the varices. There is no filling of the gastric varices but partial filling of the intrahepatic portal vein branches.

A

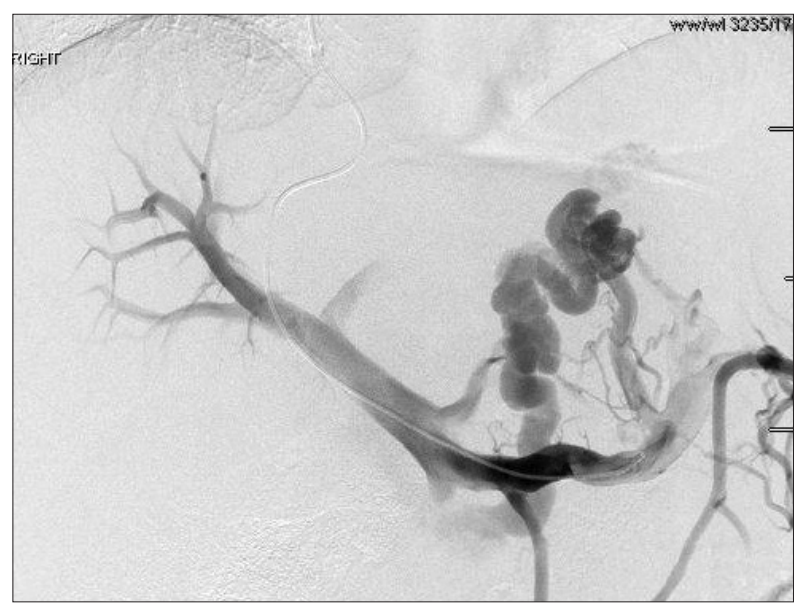

B

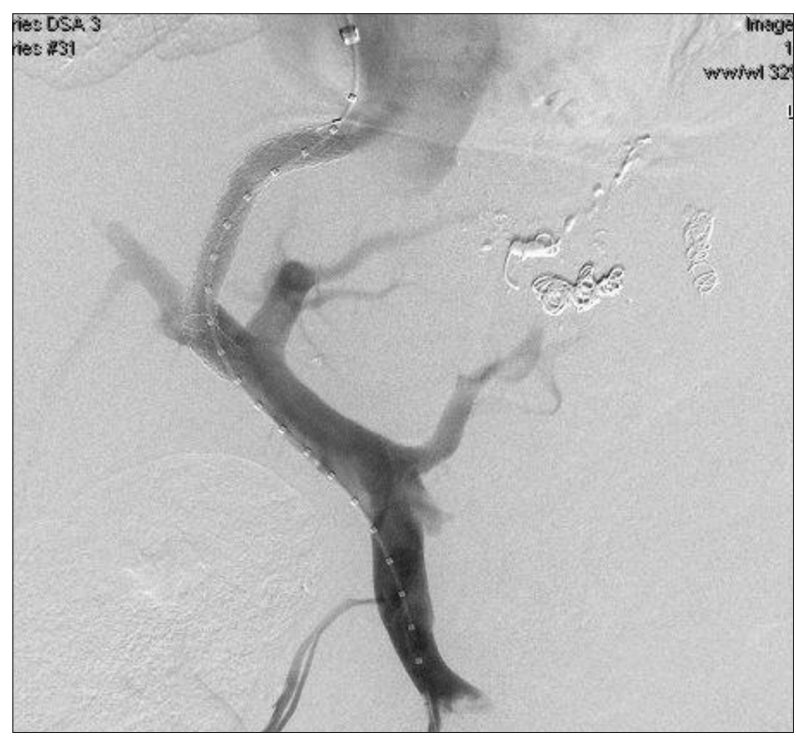


sure, an increase in spleen stiffness caused by an increase in the splenic pulp pressure should suggest TIPS dysfunction. Therefore, spleen stiffness as measured by elastography may be used for surveillance of TIPS function.

We found that splenic elasticity changed after TIPS placement in all patients who had undergone TIPS procedures. However, there was no close correlation between the spleen stiffness and portal pressure before and after TIPS placement. This finding was in line with the previous observations that there is no clear, consistent relationship between the splenic pulp pressure and the degree of reversed blood flow or collateralization and the lack of a relationship between the spleen size and the degree of esophageal varices. ${ }^{7}$ The stiffness of the spleen is variable and related to the degree of congestion as well as to the

Figure 5. Spleen stiffness versus pre-TIPS portal pressure. No measurable correlation between spleen stiffness and portal venous pressure was found.

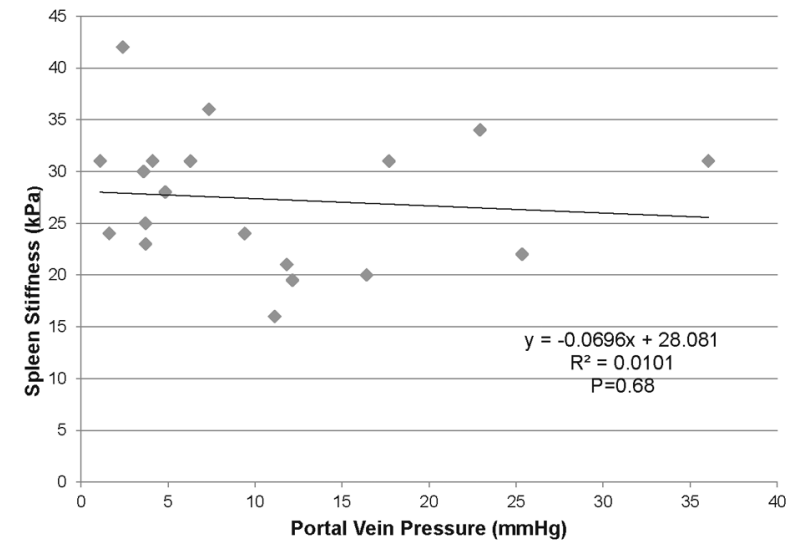

Figure 6. Pressure gradient versus median modulus after TIPS placement (18 cases). No significant correlation between the portosystemic pressure gradient and median modulus changes was found after TIPS placement.

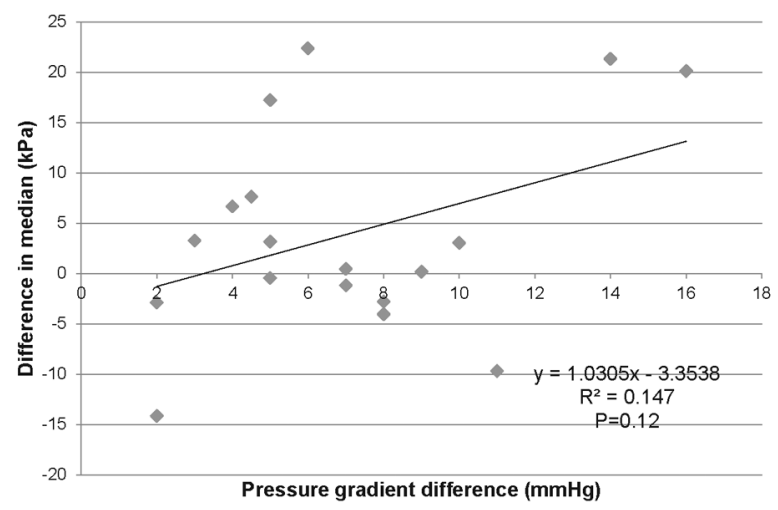

degree of fibrotic changes within the cellular architecture.

We have also found that the spleen stiffness is altered after TIPS placement, and this change can be a useful surrogate for evaluating functional changes in the TIPS. The change in spleen stiffness, whether it becomes harder or softer, may be used to establish a baseline for further evaluation. In our study, changes in the spleen stiffness did not correlate with changes in the portosystemic gradient or with the direct portal pressures. This finding contradicts earlier published reports that demonstrated a close correlation between the spleen stiffness and portal venous pressures. These previous studies did not describe embolization of any competitive shunt. ${ }^{8}$

Our study highlights the importance of expansion of the collateral circulation in these patients. Spontaneous shunts refer to the presence of spontaneous splenorenal shunts and patent paraumbilical veins. ${ }^{9,10}$ The paraumbilical vein collateral runs along the falciform ligament, distributing blood flow into the superior and inferior epigastric veins. The splenorenal shunt decompresses the portal system via a left renal vein-inferior vena cava route. The flow in these decompressing collateral pathways likely exceeded the flow though the 10-mm TIPS, which con-

Figure 7. Patients with thrombosed competitive shunts $(n=5)$ versus those with no competitive shunts $(n=14)$. There was a statistically significant change in the spleen modulus after TIPS placement between the group with competitive shunts that were either embolized or spontaneously thrombosed and the group without shunts.

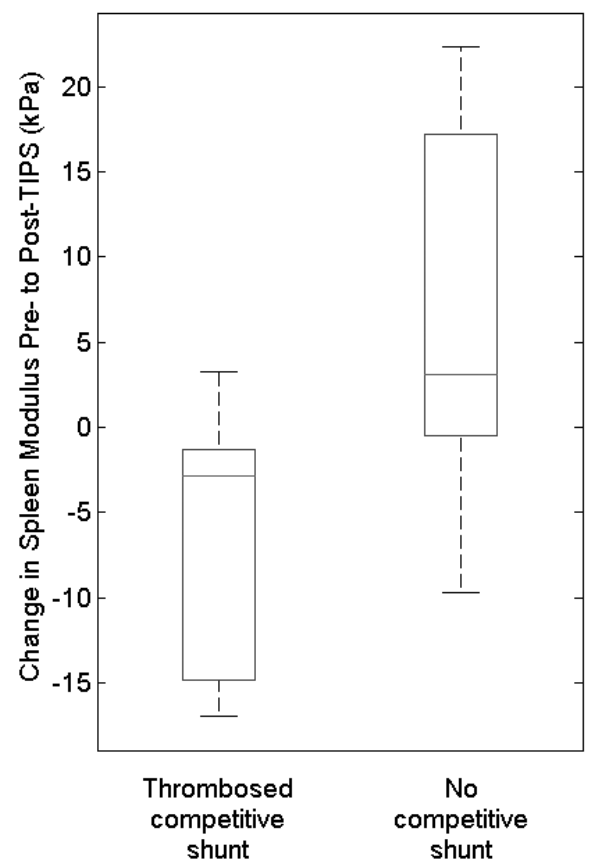


tributed to an increase in splenic congestion. The TIPS may effectively decrease the portal venous pressure and variceal filling with increased stiffness in the spleen. In addition, hemodynamically important elevations of left-sided portal pressures may persist in patients who have these shunts embolized. All patients had a reduction in the portosystemic gradient to less than $12 \mathrm{~mm} \mathrm{Hg}$, which is considered to decrease the risk of esophageal variceal bleeding. ${ }^{11}$

Immediately after TIPS placement and within several days, the stiffness of the spleen does not alter as the portal pressure changes; rather, the stiffness of the spleen changes when the portal circuit changes. This occurrence is not surprising, as after whole-graft liver transplantation, portal blood flow remains elevated for months despite normalization of portal venous pressures and normal intrahepatic resistance to flow. ${ }^{12,13}$ This factor is thought to be related to the persistence of splenomegaly and portosystemic collaterals.

Embolization of competitive collateral shunts is done to augment hepatopetal portal venous blood flow, with the theoretic advantage of decreasing the likelihood of chronic hepatic encephalopathy ${ }^{14}$ Our study highlights a possible disadvantage of this technique: the increase in left-sided portal pressures due to the obliteration of decompressive pathways, which may be necessary to prevent variceal bleeding.

The limitations of this study included the relatively noisy data. We think that this variability reflects the naturally inhomogeneous nature of the spleen. Previous studies have addressed some confounding factors affecting the reliability of acoustic radiation force impulse technology in the liver. ${ }^{15,16}$ The possibility of a tissue-related density elevation from transducer-mediated compression in subcostal and superficial measurements was described. Our intercostal approach should eliminate this factor. These studies also described differences in velocities obtained in superficial $(3.5 \mathrm{~cm}$ from the probe) or deep $(5.5 \mathrm{~cm}$ from the probe) locations. Our data were recorded from measurements at 3 to $6 \mathrm{~cm}$ from the transducer. The reproducibility of velocity measurements related to superficial or deep locations was not addressed for the spleen in these studies. The study by Kaminuma et al ${ }^{16}$ did show that the velocities recorded in the spleen did not differ before and after food consumption. We did not require that patients receive nothing by mouth for day 1 to 3 measurements.

We measured the variables over a very short period while the patients remained hospitalized for 1 to 3 days. It would be interesting to note how the spleen stiffness changes over a longer period. Spleen stiffness changes related to vascular engorgement are immediate, as hemodynamic changes in the portal venous circuit occur immediately after TIPS placement. This effect is seen angiographically in decreased collateral flow or flow that changes from hepatofugal to hepatopetal. Doppler studies have demonstrated an immediately increased main portal vein flow volume and portal vein diameter after TIPS placement. ${ }^{17}$

Finally, we cannot explain why an additional 4 patients had increased spleen stiffness after TIPS placement. No subset analysis was done based on the etiology of cirrhosis or indication for TIPS placement due to the small sample size. The fibrotic architecture, and hence the stiffness of the liver, would not be expected to change after TIPS placement; therefore, we did not obtain additional measurements in the liver.

In summary, spleen stiffness changes after TIPS placement, and this change may be useful as a predictive marker of the status of the TIPS. Spleen stiffness after TIPS placement establishes a new baseline measure of a functioning TIPS. The results also demonstrate the complexity of the portal venous circuit and can explain why splenic pulp pressures vary widely in patients with portal hypertension. Spleen stiffness decreases (softer) secondary to decreased portal pressure after TIPS placement. However, when the competitive internal shunts are embolized, spleen stiffness increases (stiffer) despite lowered portal pressure after TIPS placement. This finding suggests a potential risk of recurrent variceal bleeding with an unknown beneficial effect of embolization on TIPS function and the risk of encephalopathy. Sonographic measurement of spleen stiffness may be used for surveillance of TIPS. A further clinical study is warranted.

\section{References}

1. Zakim D, Boyer TD (eds). Hepatology: A Textbook of Liver Disease. Philadelphia, PA: WB Saunders Co; 1996

2. Baker K, Kerlan R. Imaging of portal hypertension. In: Portal Hypertension: Diagnosis and Interventions. SCVIR Syllabus. Fairfax, VA: Society of Interventional Radiology; 2001:11-43.

3. Keiding S, Solvig J, Grønbaek H, Vilstrup H. Combined liver vein and spleen pulp pressure measurements in patients with portal and splenic vein thrombosis. Scand J Gastroenterol 2004; 39:594-599.

4. Palmeri ML, Nightingale KR. Acoustic radiation force-based elasticity imaging methods. Interface Focus 201 1; 1:553-564.

5. Boyer TD, Haskal ZJ. American Association for the Study of Liver Diseases Practice Guidelines: the role of transjugular intrahepatic portosystemic shunt creation in the management of portal hypertension. Hepatology 2005; 41:386-400.

6. Darcy M. Evaluation and management of transjugular intrahepatic portosystemic shunts. AJR Am J Roentgenol 2012; 199:730-736.

7. Westaby S, Wilkinson SP, Warren R, Williams R. Spleen size and portal hypertension in cirrhosis. Digestion 1978; 17:63-68. 
8. GaoJ, Ran HT, Ye XP, Zheng YY,Zhang DZ, WangZG. The stiffness of the liver and spleen on ARFI Imaging pre and post TIPS placement: a preliminary observation. Clin Imaging 2012; 36:135-141.

9. Wind P, Alves A, Chevallier JM, et al. Anatomy of spontaneous splenorenal and gastrorenal venous anastomoses: review of the literature. Surg Radiol Anat 1998; 20:129-134.

10. Kondo T, Maruyama H, Sekimoto T, et al. Influence of paraumbilical vein patency on the portal hemodynamics of patients with cirrhosis. J Clin Gastroenterol 2014; 48:178-183.

11. Viallet A, Marleau D, Huet M, et al.Hemodynamic evaluation of patients with intrahepatic portal hypertension: relationship between bleeding varices and the portohepatic gradient. Gastroenterology 1975; 69:12971300.

12. Bolognesi M, Sacerdoti D, Bombonato G, et al. Change in portal flow after liver transplantation: effect on hepatic arterial resistance indices and role of spleen size. Hepatology 2002; 35:601-608.

13. Hadengue A, Lebrec D, Moreau R, et al. Persistence of systemic and splanchnic hyperkinetic circulation in liver transplant patients. Hepatology 1993; 17:175-178.

14. Chen S, Li X, Wei B, et al. Recurrent variceal bleeding and shunt patency: prospective randomized controlled trial of transjugular intrahepatic portosystemic shunt alone or combined with coronary vein embolization. Radiology 2013; 268:900-906

15. D'Onofrio M, Gallotti A, Mucelli RP. Tissue quantification with acoustic radiation force impulse imaging: measurement repeatability and normal values in the healthy liver. AJR Am J Roentgenol 2010; 195:132-136.

16. Kaminuma C, Tsushima Y, Matsumoto N, Kurabayashi T, TaketomiTakahashi A, Endo K. Reliable measurement procedure of virtual touch tissue quantification with acoustic radiation force impulse imaging. JUltrasound Med 2011; 30:745-751.

17. Lafortune M, Martinet JP, Denys A, et al. Short- and long-term hemodynamic effects of transjugular intrahepatic portosytemic shunts: a Doppler/manometric correlative study. AJR Am J Roentgenol 1995; 164 : 997-1002. 